\title{
NUEVOS RECURSOS TECNOLÓGICOS PARA TRABAJAR EN UN AULA DE EDUCACIÓN INFANTIL: EL CUENTO INTERACTIVO CONSIDERADO UN RECURSO DE APRENDIZAJE
}

\author{
Juana Celia Domínguez \\ José Manuel Aguilar-Parra \\ Juan Miguel Fernández-Campoy \\ $M^{a}$ Carmen Lozano Segura \\ Departamento de Psicología Evolutiva y de la Educación, Universidad de Almería. \\ E-mail: jmaguilar@ual.es
}

https://doi.org/10.17060/ijodaep.2017.n1.v3.1013

Fecha de Recepción: 2 Enero 2017

Fecha de Admisión: 1 Abril 2017

\section{RESUMEN}

Actualmente, las nuevas Tecnologías de la Información y Comunicación (TIC) se consideran un recurso clave para la enseñanza, de tal manera que este concepto está evolucionando y tenemos un nuevo término: TAC (Tecnologías del Aprendizaje y la Comunicación). Esta comunicación analiza algunos de los recursos que pueden ser utilizados e integrados en aulas de Educación Infantil, en concreto los cuentos interactivos, ya que según la normativa actualdebemos introducirlas durante esta etapa. Con el fin de integrar los recursos tecnológicos en el ámbito escolar, se debe formar al profesorado para que adquiera competencias tecnológicas, innove y experimente en el aula junto a su alumnado.

Palabras claves: TIC, recursos tecnológicos, Ed.Infantil, cuento interactivo.

\section{ABSTRACT}

New technological resources to work in a classroom of Pre - school Education: the interactive story is considered a learning resource.

Currently, the new Information and Communication Technologies(ICT) are considered a key resource for education. This concept is progressing and we have a new term: LCT (Learning and Communication Technologies). This communication analyzes some resources which can be used and integrated in classrooms of pre - school education, in particular the interactive story, because according to the current regulations, we must introduce it during this period. With the purpose of integrating technological resources in the school area, we must educate teachers to get technological competences, innovate and experiment in classrooms together with their student body.

Key words: ICT, technological resources, pre - school education, interactive story. 


\section{NUEVOS RECURSOS TECNOLÓGICOS PARA TRABAJAR EN UN AULA DE EDUCACIÓN INFANTIL: EL CUENTO INTERACTIVO CONSIDERADO UN RECURSO DE APRENDIZAJE}

\section{ANTECEDENTES DE LA TEMÁTICA A TRATAR.}

La introducción de las tecnologías de la información y la comunicación ha implicado grandes cambios en la sociedad, la cual es el resultado de la fuerte influencias que éstas ejercen sobre las personas.

Se considera que sus orígenes residen en la época de los años 50 , periodo a partir del cual empezaron a evolucionar hasta llegar a la era de los "nativos e inmigrantes digitales", término acuñado por Prensky (2011).

La etapa de educación infantil ha integrado en su currículo este tipo de tecnologías dando uso a diferentes recursos como son el ordenador, el proyector, pizarra digital, etc. incluso, actualmente,algunos colegios públicos y privados teniendo en posesión una tableta o portátil individual con el que desarrollar sus actividades curriculares, donde, según Cacheiro, Sánchez y González (2015), el profesor adquiere un papel de educador social como investigador en el aula.

Los distintos recursos TIC deben abordarse desde una perspectiva organizativa y didáctica. Algunos de los que se vienen dando en estos últimos años hasta hoy son: e - learning, web 2.0 y 3.0, redes sociales, videojuegos, cuentos interactivos, recursos móviles, etc. (Sánchez, 2016). Su importancia no reside solamente en el uso y manejo de estos recursos, sino también en que el alumno adquiera la capacidad de realizar una selección crítica de la información que nos ofrecen las nuevas tecnologías.

Los recursos tecnológicos deben estar integrados en procesos formativos con unos objetivos educativos muy bien definidos, con una adecuada planificación de actividades y con el diseño de una evaluación de los aprendizajes que sea coherente con la metodología por la que hayamos optado (Gisbert y Holgado, 2011).

Como sugieren Robinson y Aronica (2016), es imprescindible aprovechar el potencial de los recursos tecnológicos disponibles fomentando de esta manera la participación de los estudiantes para que pierdan el miedo a equivocarse, desarrollen su pasión por aprender y su creatividad, y estén preparados para afrontar los retos que les depare el futuro.

Esta comunicación se centrará en valorar el cuento interactivo como un recurso de aprendizaje ya que, como indica Educación 3.0 (2016), en ellos combinan textos y elementos visuales, así como ejercicios y actividades para que los alumnos se impliquen en las tramas narradas. Por ello, pondré en práctica un cuento interactivo en un aula de Educación Infantil titulado "Desaburrimiento" (Omnis Cellula, n.d.)., el cual pertenece a la página web "ClicClicClic: cuentos interactivos" (www.cuentosinteractivos.org/), dividida en dos bloques: uno que comprende las edades de entre 3-6 años, Ilamado "Iván y Navi, el poder de la imaginación", que es el escogido para la realización de la investigación y un segundo bloque destinado para el alumnado de entre 6-8 años.

\section{OBJETIVOS DE LA INVESTIGACIÓN.}

Los objetivos que me propongo se orientan por dos vertientes: una referida a la práctica docente, cuyo objetivo general sería:

Incorporar los nuevos recursos tecnológicos para trabajar con ellos en el aula de Educación Infantil.

La otra vertiente hace referencia a la perspectiva didáctica, por un lado referida al área de matemáticas:

Reconocer distintas figuras geométricas y los números que aparecen en un cuento interactivo.

Por otro lado, referida al lenguaje:

Conocer la grafía de diversas vocales y consonantes.

Aunque esta investigación se centra en estas áreas, el aprendizaje será globalizado e trabajaremos las tres áreas del currículo trasversalmente durante el trascurso del cuento interactivo. 


\section{MUESTRA Y/O PARTICIPANTES.}

La investigación ha sido realizada en el C.E.I.P. "San Gabriel” (Almería), en una clase de $2^{0}$ ciclo de Educación Infantil en el nivel de 4 años, compuesta por 24 alumnos/as, de los cuales 11 son niñas y 13 son niños. Los estudiantes presentan un nivel de conocimientos homogéneo y adquieren los aprendizajes adecuadamente. En el caso de que alguno de los alumnos presentara Necesidades Específicas de Apoyo Educativo (NEAE), le realizaría las adaptaciones necesarias con el fin de ofrecerle una respuesta educativa ajustada a sus necesidades, de este modo, mi acción docente será llevada a cabo bajo el principio de atención a la diversidad.

\section{METODOLOGÍA E INSTRUMENTOS UTILIZADOS.}

He optado por desarrollar una metodología de investigación observacional, en la que el investigador realiza una observación directa del participante. Esta se caracterizará por ser de carácter mixto(cualitativa y cuantitativa), y basada en los principios de participación, globalización, socialización, atención a la diversidad, aprendizaje significativo y trabajo cooperativo. Además se pone en prácticarealizando una evaluación inicial de la competencia curricular del alumnado para partir de sus conocimientos previos y poder realizar así aprendizajes significativos(Ausubel, 2002).

Los materiales que empleo son un ordenador, una pizarra digital, un proyector, plastilina, palillos, distintos objetos de la clase y un bloc de notas de campo.

\subsection{Descripción metodología de investigación.}

En la actividad preparatoria organizaré a los alumnos/as sentados en sus sitios en pequeños grupos (están sentados por pequeños grupos durante su rutina diaria). En esta actividad diré a cada alumno que me diga algún objeto en la clase que tenga por ejemplo forma cuadrada (también incluiré la forma rectangular, triangular o circular), después de las formas geométricas trabajaré los números (fijándome sobre todo en el aprendizaje del 1 al 6) indicándoles que cada equipo me busque por ejemplo 4 lápices, 2 gomas, etc., y por último, de nuevo en sus sitios les pediré que me digan palabras que empiecen por las vocales "U", "A", "I", "O" y "E". Todos estos pequeños juegos tendrán una duración aproximada de 10 min entre las 3 actividades.

En la actividad central dispondré a los alumnos en el corcho de la asamblea, de este modo todos los niños tendrán una visión directa hacia la pizarra digital. Comenzaremos a trabajar con el cuento llevando a cabo las distintas actividades que nos propone. A continuación, detallaré en el siguiente cuadro el desarrollo: 
NUEVOS RECURSOS TECNOLÓGICOS PARA TRABAJAR EN UN AULA DE EDUCACIÓN INFANTIL: EL CUENTO INTERACTIVO CONSIDERADO UN RECURSO DE APRENDIZAJE

Cuadro objetivos, contenidos, explicación de la actividad justificación de objetivos de las escenas del cuento.

\begin{tabular}{|c|c|c|c|c|}
\hline ESCENA & OBJETIVOS & CONTENIDOS & ACTIVIDAD & OBJETIVOS \\
\hline \multirow[t]{3}{*}{$\mathbf{N}^{0} 1}$. & \multicolumn{2}{|l|}{ MATEMÁTICAS } & \multirow{3}{*}{$\begin{array}{l}\text { Encontrar la } \\
\text { llave roja. }\end{array}$} & \multirow{3}{*}{$\begin{array}{l}\text { Representación } \\
\text { del número } 4 \text { en } \\
4 \text { llaves. }\end{array}$} \\
\hline & $\begin{array}{l}\text {-Reconocer los } \\
\text { números del 1-4 }\end{array}$ & $\begin{array}{l}\text {-Números del } 1 \\
\text { al } 4 \text { (llaves) }\end{array}$ & & \\
\hline & \multicolumn{2}{|l|}{ LENGUA } & & \\
\hline \multirow[t]{4}{*}{$\mathbf{N}^{0} 2}$. & \multicolumn{2}{|l|}{ MATEMÁTICAS } & \multirow{4}{*}{$\begin{array}{l}\text { Hacer clic en } \\
\text { el cofre. }\end{array}$} & \multirow{3}{*}{$\begin{array}{l}\text { Visualización de } \\
\text { la palabra } \\
\text { desaburrimiento }\end{array}$} \\
\hline & -Conocer el $n^{\circ} 2$ & -Número 2 & & \\
\hline & \multicolumn{2}{|l|}{ LENGUA } & & \\
\hline & $\begin{array}{l}\text {-Identificar las } \\
\text { letras de la palabra } \\
\text { "desaburrimiento } \\
\text { „. }\end{array}$ & $\begin{array}{l}\text {-Letras } \\
\text { "desaburrimient } \\
o "\end{array}$ & & $\begin{array}{l}\text { en mayúscula, } \\
\text { representación } \\
\text { de } 2 \text { niños en la } \\
\text { escena. }\end{array}$ \\
\hline \multirow{4}{*}{$\begin{array}{l}N^{0} 3,4,5, \\
6 \text { y } 7 .\end{array}$} & \multicolumn{2}{|l|}{ MATEMATICAS } & \multirow{4}{*}{$\begin{array}{l}\text { Hacer clic en } \\
\text { Iván y Navi y } \\
\text { por último en } \\
\text { adelante. }\end{array}$} & \multirow{4}{*}{$\begin{array}{l}\text { Total de } \\
\text { animales que } \\
\text { salen en la } \\
\text { actividad, y los } \\
\text { nombres de } \\
\text { dichos animales. }\end{array}$} \\
\hline & $\begin{array}{l}- \text { Reconocer } \mathrm{n}^{\circ} 1 \mathrm{y} \\
3 \text {. }\end{array}$ & $-\mathrm{N}^{\mathrm{o}} 1$ y 3 & & \\
\hline & \multicolumn{2}{|l|}{ LENGUA } & & \\
\hline & $\begin{array}{l}\text {-Conocer las letras } \\
\text { "o", "e" y "d". }\end{array}$ & $\begin{array}{l}\text {-Letra inicial de } \\
\text { los animales: "o", } \\
\text { "e" y "d". }\end{array}$ & & \\
\hline $\mathbf{N}^{\mathbf{0}} \mathbf{8}, \mathbf{9}$, & \multicolumn{2}{|l|}{ MATEMÁTICAS } & Buscar & Identificación en \\
\hline
\end{tabular}




\begin{tabular}{|c|c|c|c|c|}
\hline $\begin{array}{ll}10,11 y \\
12 . & \end{array}$ & $\begin{array}{l}\text {-Reconocer } \mathrm{n}^{\circ} 1 \text { y } \\
3 . \\
\text {-Discriminar las } \\
\text { horas del reloj. } \\
\text { - Identificar } \\
\text { formas } \\
\text { geométricas. }\end{array}$ & $\begin{array}{l}-\mathrm{N}^{\mathrm{o}} 3 \text { (ratones) } \mathrm{y} \\
\mathrm{n}^{\mathrm{o}} 1 \text { (un gusano, } \\
\text { una mujer) } \\
- \text { Horas del reloj: } \\
1-12 \quad \text { (solo } \\
\text { evaluable del } 1 \text { al } \\
6 \text { ). } \\
\text {-Formas } \\
\text { geométricas: } \\
\text { círculo, } \\
\text { rectángulo, } \\
\text { triángulo. }\end{array}$ & $\begin{array}{l}\text { esconden las } \\
\text { señales de } \\
\text { tráfico y hacer } \\
\text { clic en el } \\
\text { autobús. } \\
\text { Comentar las } \\
\text { horas del reloj. }\end{array}$ & $\begin{array}{l}\text { la imagen de las } \\
\text { figuras } \\
\text { nombradas y } \\
\text { representación } \\
\text { de los números } \\
\text { en elementos. }\end{array}$ \\
\hline & LENGUA & & & \\
\hline $\begin{array}{l}\mathbf{N}^{0} 13,14 \\
\text { y } 15 .\end{array}$ & $\begin{array}{l}\text {-Discriminar } \\
\text { formas } \\
\text { geométricas. } \\
\text {-Reconocer el } \mathrm{n}^{\circ} \\
6 .\end{array}$ & $\begin{array}{l}\text {-Formas } \\
\text { geométricas: } \\
\text { óvalo, } \\
\text { rectángulo, } \\
\text { cuadrado, } \\
\text { estrella, triángulo } \\
\text { y círculo (sólo } \\
\text { evaluables } \\
\text { círculo, triángulo } \\
\text { y rectángulo). } \\
\text {-No } 6 \text { (número de }\end{array}$ & $\begin{array}{l}\text { Hacer clic en } \\
\text { el cangrejo, } \\
\text { poner cada } \\
\text { forma en el } \\
\text { lugar } \\
\text { adecuado, } \\
\text { pinchar en el } \\
\text { pájaro. }\end{array}$ & $\begin{array}{l}\text { Representación } \\
\text { en la escena de } \\
\text { las formas } \\
\text { geométricas y } \\
\text { objetos citados } \\
\text { y el } n^{\circ} 6 \text { por el } \\
n^{\text {o total de }} \\
\text { formas } \\
\text { observadas. }\end{array}$ \\
\hline
\end{tabular}




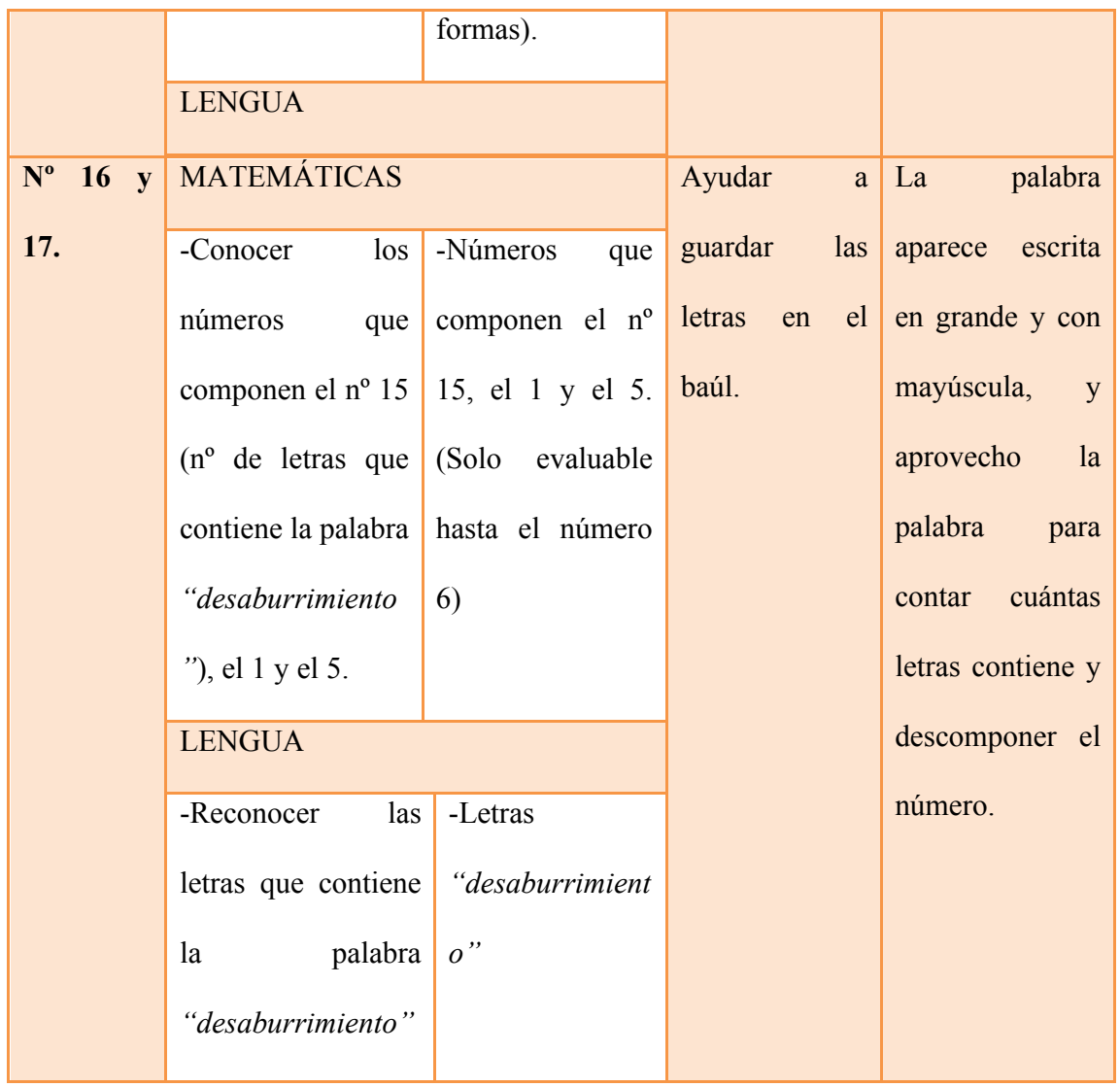

La actividad de explotación consistirá en jugar con plastilina y con palillos de madera sentados en sus sitios trabajando individualmente, con estos materiales deberán de construir formar geométricas, números y letras mediante la unión de los palillos utilizando como nexo la plastilina. Cada alumno/a tendrá que elaborar una letra, un número y una forma geométrica, una vez que hayan terminado me pasaré por las mesas preguntando que ha hecho cada uno/a.

Además de los contenidos mencionados anteriormente, también trabajaré otra serie de conceptos, los cuales no serán objeto de esta investigación pero formarán parte del desarrollo de la sesión para que el alumnado no se sienta distanciado de los contenidos que están viendo en la unidad llevada a cabo por su tutora. 


\begin{tabular}{|c|c|c|}
\hline ESCENAS & OBJETIVOS & CONTENIDOS \\
\hline \multirow{3}{*}{$\mathbf{N}^{0} 1}$. & \multicolumn{2}{|l|}{ MATEMÁTICAS } \\
\hline & \multicolumn{2}{|l|}{ LENGUA } \\
\hline & $\begin{array}{l}\text {-Discriminar los colores rojo, verde, } \\
\text { azul y gris (blanco y negro) } \\
\text {-Conocer el concepto cerrado. }\end{array}$ & $\begin{array}{l}\text {-Percepción de los colores (llaves) } \\
\text {-Concepto cerrado (cofre) } \\
\text {-Controversia: guardar. }\end{array}$ \\
\hline \multirow[t]{4}{*}{$\mathbf{N}^{0} 2}$. & \multicolumn{2}{|l|}{ MATEMÁTICAS } \\
\hline & -Distinguir entre lleno y vacío. & Concepto Lleno - vacío \\
\hline & \multicolumn{2}{|l|}{ LENGUA } \\
\hline & -Conocer el concepto "abierto". & -Concepto abierto (cofre) \\
\hline \multirow{3}{*}{$\begin{array}{l}N^{0} 3,4,5, \\
6 \text { y } 7 .\end{array}$} & \multicolumn{2}{|l|}{ MATEMATICAS } \\
\hline & -Distinguir entre mucho - poco. & -¿Concepto mucho (Miles de animales)? \\
\hline & \multicolumn{2}{|l|}{ LENGUA } \\
\hline \multirow{3}{*}{$\begin{array}{l}N^{0} 8,9,10, \\
11 \text { y } 12 .\end{array}$} & \multicolumn{2}{|l|}{ MATEMÁTICAS } \\
\hline & \multicolumn{2}{|l|}{ LENGUA } \\
\hline & $\begin{array}{l}\text {-Reconocer trazos curvos, } \\
\text { horizontales y verticales. }\end{array}$ & $\begin{array}{l}\text {-Trazos curvos, horizontales y verticales } \\
\text { (formas geométricas). } \\
\text {-Educación vial: respeto por los coches, } \\
\text { comportamiento en los vehículos, } \\
\text { cuidado en la calle, }\end{array}$ \\
\hline$N^{0} 13,14 y$ & \multicolumn{2}{|l|}{ MATEMÁTICAS } \\
\hline
\end{tabular}


NUEVOS RECURSOS TECNOLÓGICOS PARA TRABAJAR EN UN AULA DE EDUCACIÓN INFANTIL: EL CUENTO INTERACTIVO CONSIDERADO UN RECURSO DE APRENDIZAJE

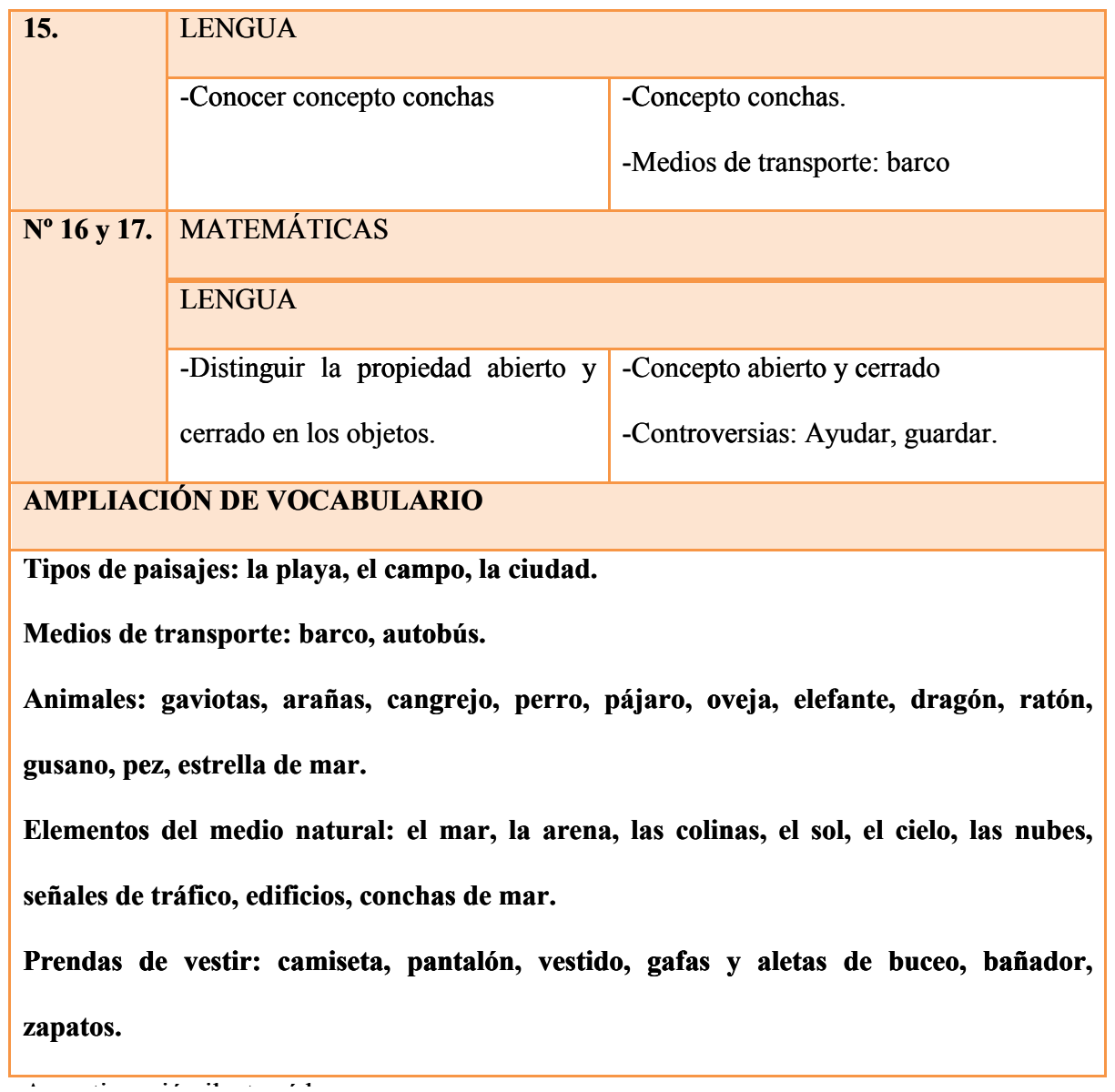

.A continuación ilustraré las escenas

\section{Escenas}

Escena 1

Escena 2
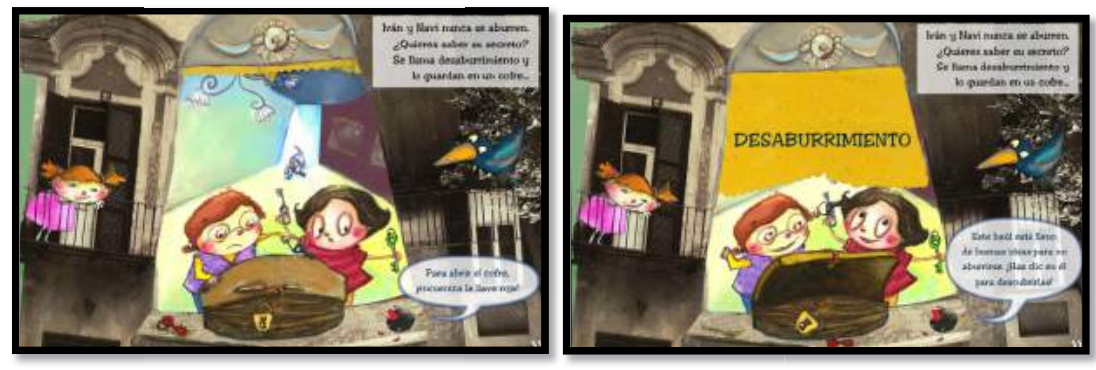
LA PSICOLOGÍA HOY: RETOS, LOGROS Y PERSPECTIVAS DE FUTURO. PSICOLOGÍA INFANTIL

Escena 3

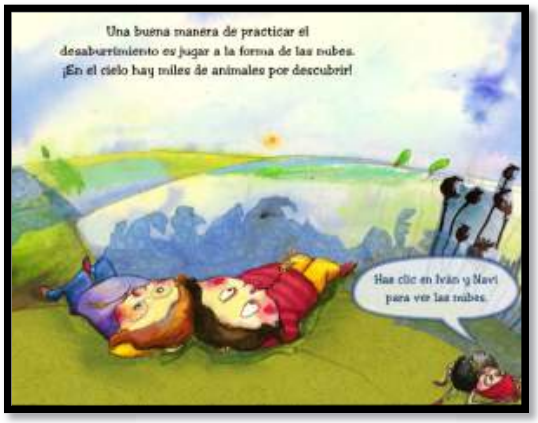

Escena 5

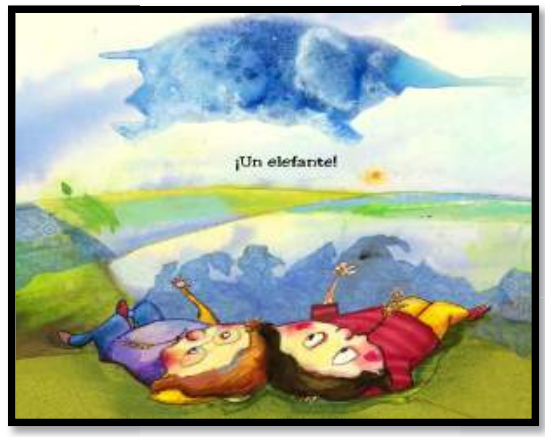

Escena 7

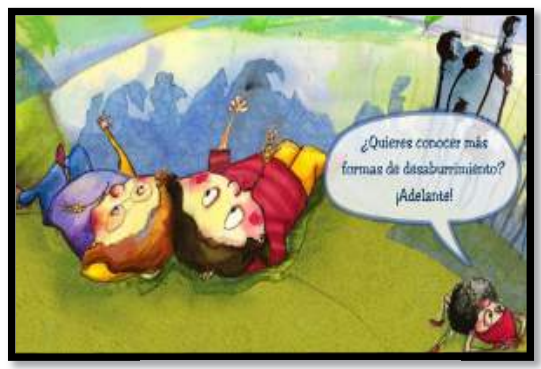

Escena 4

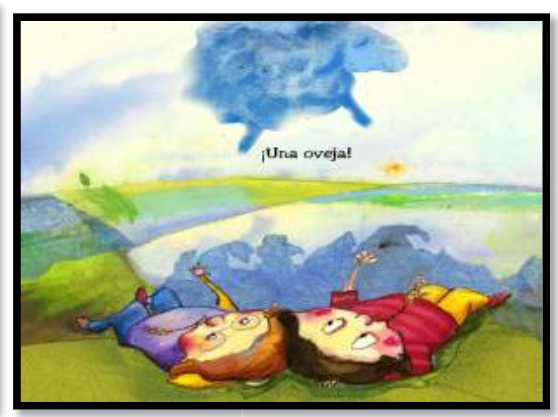

Escena 6

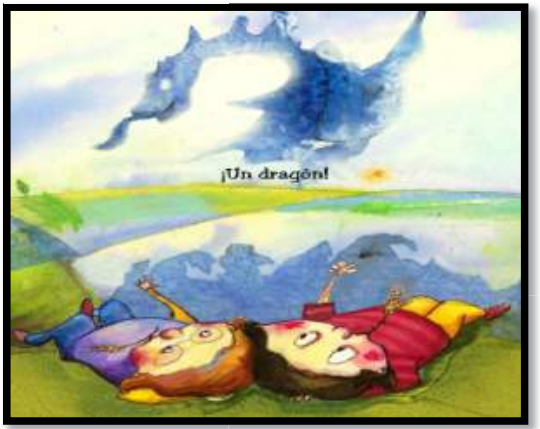

Escena 8

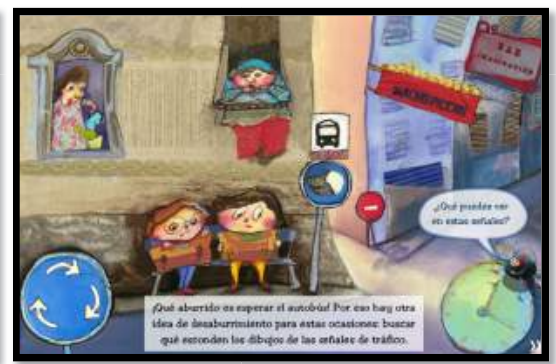


NUEVOS RECURSOS TECNOLÓGICOS PARA TRABAJAR EN UN AULA DE EDUCACIÓN INFANTIL: EL CUENTO INTERACTIVO CONSIDERADO UN RECURSO DE APRENDIZAJE

Escena 9

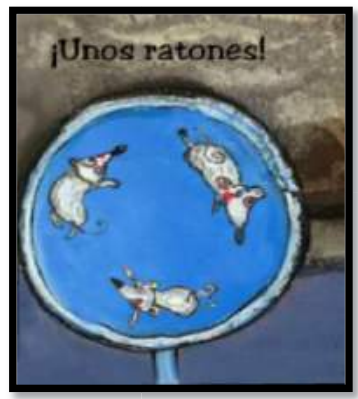

Escena 10

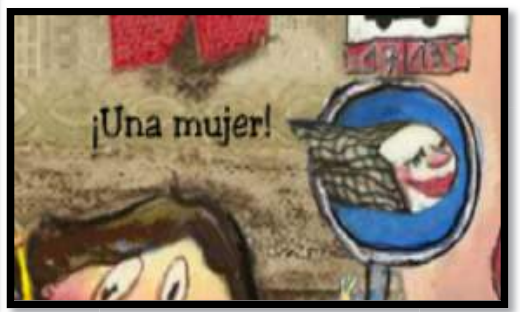

Escena 12

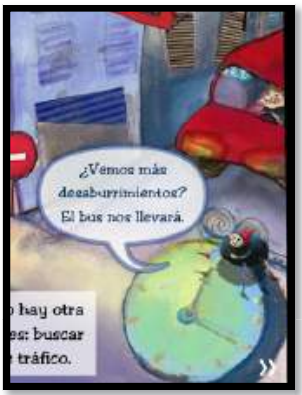

Escena 13

Escena 14
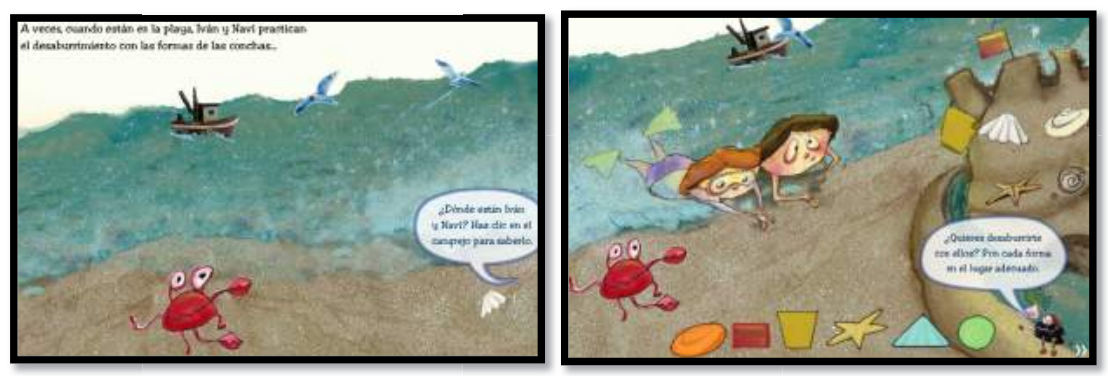

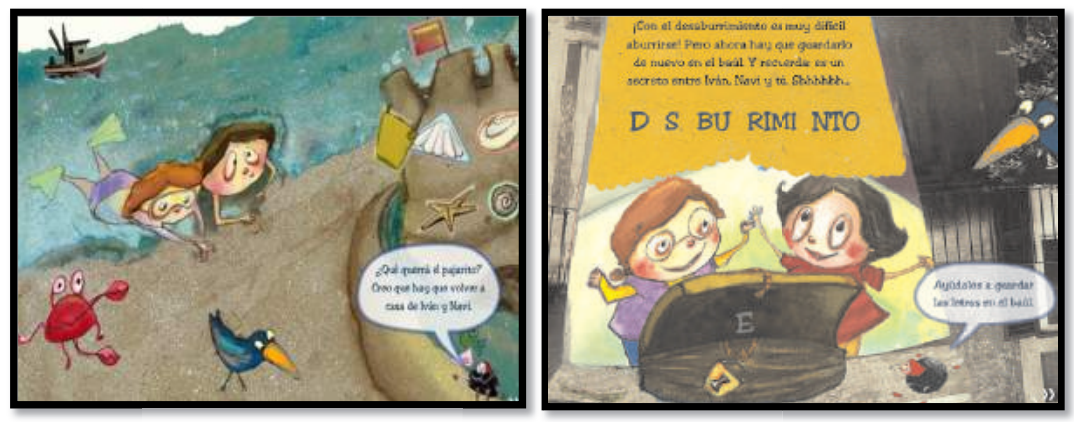

\section{Escena 17}

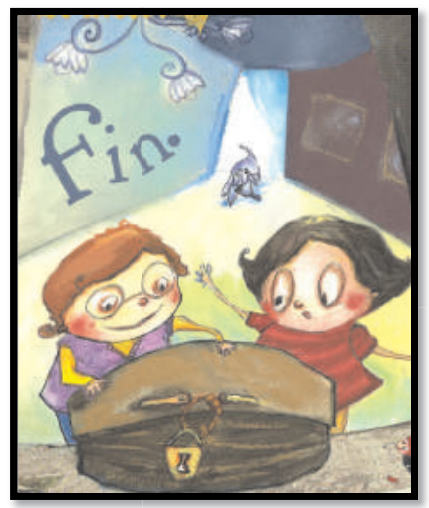

\section{RESULTADOS ALCANZADOS.}

\subsection{Recogida de datos.}

El trabajo está estructurado en tres acciones 0 actividades: la primera es una actividad preparatoria (evaluación de conocimientos previos); la segunda es una actividad central en la que introduzco los conocimientos a aprender [los números del 1 al 6 (aunque se verán más), las figuras círculo, óvalo, rectángulo, cuadrado, triángulo y estrella y las grafías de las letras de la palabra "DESABURRIMIENTO", y la tercera es una actividad de explotación que pretende poner a prueba a los niños/as para comprobar si han adquirido correctamente los contenidos.

La recogida de datos la realizo a través de un registro directo en un bloc de campo, tanto por mí (alumna en prácticas) como por la docente encargada del aula. Tras el desarrollo de cada sesión, ambas investigadoras contrastamos nuestras anotaciones y datos.

\subsection{Análisis de datos.}

Una vez recopiladas todas las anotaciones, me dispongo a analizarlas teniendo en cuenta los 


\section{NUEVOS RECURSOS TECNOLÓGICOS PARA TRABAJAR EN UN AULA DE EDUCACIÓN INFANTIL: EL CUENTO INTERACTIVO CONSIDERADO UN RECURSO DE APRENDIZAJE}

objetivos que pretendo conseguir y que son objeto de esta investigación. Durante el desarrollo de las actividades, ambas investigadoras anotaban las respuestas erróneas que tenía cada alumno así como otros datos de interés acerca de los contenidos que estábamos viendo, por ejemplo si sus conocimientos eran más o menos amplios a los que estaban establecidos en el cuento. Con posterioridad, agrupamos los datos a través de la concordancia consensuada.

Con respecto a la adquisición de los conocimientos, la siguiente gráfica muestra los valores obtenidos:

Gráfica 1:

Estudiantes que alcanzan los objetivos

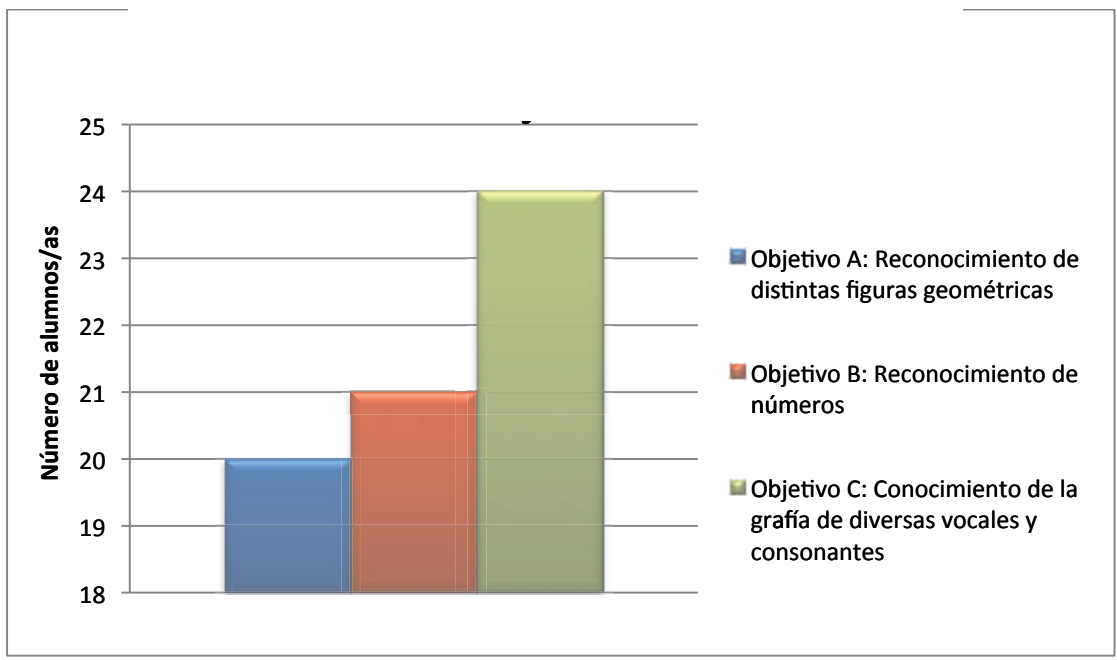

A nivel cualitativo he detectado algunos hechos notables que formarán parte del análisis de los datos obtenidos.

Varios sujetos no tienen conocimiento de la forma rectangular que se apreciaban en la imagen. (Extracto 1)

Extracto 1. Sujetos $9,11,21$ y 25 . Alumnos/ as que no conocen la forma rectangular.

Sujeto 9: círculo, triángulo y cuadrado.

Investigadora: ¿Y dónde está el rectángulo?

Sujeto 9, 11, 21 y 25: no hay seño.

Sujetos que saben asociar la forma geométrica con la forma de un objeto. (Extracto 2)

Extracto 2. Todos los sujetos, menos 9, 11, 21 y 25. Asociación de formas con objetos.

Todos menos 9, 11, 21 y 25: seño mira, el rectángulo es igual que la bandera.

Investigadora: ¿cómo que es igual?

Todos menos 9, 11, 21 y 25: si seño, que tienen la misma forma.

ídem con las demás formas.

El total de los sujetos sabe contar hasta el 6 sin cometer fallos, tres de esos alumnos/as lo logran con mi ayuda. (Extracto 3) 
Extracto3. Todos los sujetos. Ejemplo de logros y fallos al contar.

Sujeto 1: imirad cuantas letras!

Investigadora: Vamos a contarlas.

Todos los sujetos: 1, 2, 3, 4, 5, 6, 7...

Sujeto3: 1, 3, 4, 7

Sujeto $7: 2,5,3$

Sujeto 2: 1, 2, 3, 6 .

Investigadora: Vamos a empezar a contar de nuevo, yo os ayudo.

Todos los sujetos asocian las letras de la palabra "Desaburrimiento" a otras palabras que empiezan por dicha letra. (Extracto 4)

Extracto 4. Todos los sujetos. Asociación de letras con la letra inicial de palabras.

Todos los sujetos: la A de Adrián, Aitana, Aitor, Aarón y abeja.

Investigadora: ¿todas esas palabras empiezan con la A?

Todos los sujetos: sí, y con la I empieza indio.

Una vez analizados los resultados prosigo con su discusión.

\section{DISCUSIÓN.}

A continuación voy a proceder a la discusión de los resultados obtenidos. En cuanto al objetivo A, como refleja la gráfica 1, en relación al área de matemáticas un total de 20 niños/as reconocen las distintas figuras geométricas que se trabajan en el cuento (véase extracto 2) y tan solo cuatro no han reconocido la figura rectangular (véase extracto 1). Creo que estos sujetos no la han adquirido aún debido al poco tiempo que llevan estudiando esa forma en clase. A pesar de ello parece que la gran mayoría de la clase cumple el objetivo A.

Respecto al objetivo $B$ (véase gráfica 1), 21 alumnos/as conocen los números del 1 al 6 , fallando los sujetos 2, 3 y 7 como refleja el extracto 3 . Creo que no tienen adquirida la serie numérica debido a su falta de atención en clase y a su distracción cuando se le intenta explicar. Con lo cual, parece que el objetivo se cumple.

En relación al objetivo C (véase gráfica 1), correspondiente al área del lenguaje, los 24 niños/as reconocen las grafías de las letras trabajadas como se observa en el extracto 4 . Creo que la causa de este éxito es su pasión por las letras, les encantaba trabajar el abecedario y decir palabras que empezaran con cada una de las letras. Con ello parece que dicho objetivo se cumple.

A la vista de todo lo anterior, me surgen las siguientes preguntas abiertas acerca de los posibles motivos por los cuales los estudiantes no llegaron a adquirir los conocimientos, ¿será por la falta de atención?, ¿es posible que deban madurar para adquirirlos?, las cuales pueden ser objeto de una posible investigación futura.

Una vez discutidos los resultados, en el siguiente apartado trataré las conclusiones.

\section{CONCLUSIONES.}

Con todos los resultados discutidos, desde la vertiente de mi práctica docente, he podido observar que mi objetivo planteado lo he podido superar, ya que la idea de contar un cuento de manera interactiva, saliéndonos de los estándares habituales e incorporando recursos tecnológicos al aula, ha resultado ser todo un éxito. El alumnado quedaba sorprendido y entusiasmado, lo que favorece un aprendizaje lúdico basado en el juego en el que el niño es el protagonista de su proceso educativo, y la maestra me afirmó que a partir de ese momento incorporaría este tipo de recursos como parte de su programación. 


\section{NUEVOS RECURSOS TECNOLÓGICOS PARA TRABAJAR EN UN AULA DE EDUCACIÓN INFANTIL: EL CUENTO INTERACTIVO CONSIDERADO UN RECURSO DE APRENDIZAJE}

Desde la perspectiva didáctica, concluyo que los niños que no han adquirido los conocimientos tiene como causa la falta de atención ya que les gustaba mucho el decorado del cuento o incluso su desarrollo madurativo, debido a la existencia de varios infantes con edad inferior a cuatro años 0 con ellos recién cumplidos, también es posible que ese no fuera su mejor momento de concentración.

La mayoría del alumnado alcanza la totalidad de mis objetivos como detallo en la gráfica 1, y para aquellos estudiantes que no lo hayan superado tendría que volver a plantearme otras formas de aprendizaje mediante las cuales llegaran a superarlos.

A continuación mencionaré los puntos débiles y fuertes que han resultado de la investigación llevada a cabo, y seguidamente, una de las propuestas de mejora y perspectivas futuras de investigación.

En cuanto a los puntos débiles, creo que uno de ellos fue la falta de práctica de los alumnos/as en trabajar a través de las tics. Era nuevo para ellos desarrollar las clases en la asamblea contando un cuento en la pizarra digital y que fueran ellos mismos los que tenían que ayudar a seguir contando el cuento, hecho que influyó en la investigación en relación al poco contacto que habían tenido con este tipo de recursos.

Algunos de los puntos fuertes de la investigación son desarrollar la sesión a primera hora de la mañana, lo cual sirvió de mucho ya que se captó toda la atención de la muestra objeto de estudio para su mayor rendimiento, otro es el ambiente que creé en clase de tranquilidad y concentración, y por último cómo gestioné el clima del aula, intentando serenar a los niños/as más inquietos haciéndoles preguntas acerca del cuento creando intriga por saber más acerca de él.

Finalmente, una de las propuesta de mejora que establecería sería partir de un cuento que fuera del interés del alumnado y sintieran curiosidad por él, con esta opción mostrarían más interés ya que sería de su agrado, y al conocer su argumento podrían construir a partir de ahí nuevos aprendizajes. Además, yo creo que podría profundizar dicha investigación planteándome nuevos objetivos y conceptos, y como segunda perspectiva futura plantearía a tres clases de 4 años de colegios distintos que llevaran a cabo mi investigación, con el fin de contrastar y discutir sus resultados con los míos.

\section{REFERENCIAS BIBLIOGRÁFICAS SEGÚN NORMATIVA APA.}

-Ausubel, D. (2002). Adquisición y retención del conocimiento. Una perspectiva cognitiva. Barcelona: Paidós.

-Cacheiro, M., Sánchez, C. y González, J. M. (2015). Recursos tecnológicos en contextos educativos. Madrid: UNED.

-Educación 3.0. (13/06/2016). Las mejores apps de cuentos interactivos. Recuperado de http://www.educaciontrespuntocero.com/recursos/familias-2/las-mejores-apps-de-cuentosinteractivos/24116.html

-Gisbert, M. y Holgado, J. (2011). Las herramientas tecnológicas como apoyo al proceso de enseñanza-aprendizaje: La pizarra digital interactiva. En prensa.

-Asociación Omnis Cellula, (n.d.). ClicClicClic. Cuentos interactivos. Recuperado de http://www.cuentosinteractivos.org/

-Prensky, M. (2011). Enseñar a nativos digitales. Madrid: SM

-Sánchez, J. (2016). Tecnologías de la comunicación y la información aplicadas a la educación. Madrid: Síntesis. 